\title{
Irregular menstrual cycles in a young woman
}

\author{
Jacqueline A. Boyle PhD, Helena J. Teede PhD
}

Competing interests: None declared.

This article has been peer reviewed.

\section{Correspondence to:}

Jacqueline Boyle, jacqueline.boyle@monash .edu

CMAJ 2014. DOI:10.1503 /cmaj.130667
A 28-year-old woman reports irregular menstruation since she stopped taking an oral contraceptive pill three years earlier. Menarche occurred at 14 years of age, and her menstrual cycles had been regular before she started the oral contraceptive pill for contraception at age 16. She has gained $15 \mathrm{~kg}$ since age 16 and has hair growth on her top lip, which she waxes. She is planning to conceive a child in the next two years.

\section{Does this woman have polycystic ovary syndrome?}

The most likely diagnosis is polycystic ovary syndrome, which is the most common endocrine condition among women of reproductive age. It affects $12 \%-21 \%$ of women and increases in frequency with increasing weight. ${ }^{1,2}$ The differential diagnoses to consider for this patient include hyperprolactinemia, thyroid dysfunction and, rarely, nonclassic congenital adrenal hyperplasia, Cushing syndrome and virilizing tumours. ${ }^{1,3}$

\section{What questions should be asked and what should be looked for on clinical examination?}

Diagnosis of polycystic ovary syndrome using the Rotterdam criteria requires the presence of two of the following symptoms: oligo-ovulation or anovulation, hyperandrogenism (clinical or biochemical) and polycystic ovaries on ultrasonography. ${ }^{3}$

The following questions and features may assist in establishing a diagnosis:

- What is the patient's menstrual cycle pattern? Cycles shorter than 21 or longer than 35 days are likely to be anovulatory. ${ }^{1}$

- Is there excess unwanted body or facial hair? If so, for how long and how has it progressed over time? What treatment is used? Is there hair loss on the scalp?

- Is there a family history of infertility, polycystic ovary syndrome or diabetes?
Because both thyroid dysfunction and hyperprolactinemia can cause cycle irregularity, questions regarding typical symptoms (e.g., galactorrhea, lethargy, recent changes in weight) of these disorders may be helpful. However, the symptoms can be insidious.

For other causes of cycle irregularity and hirsutism, which are rare, history and examination should suffice in most cases without the need for additional investigation. Questioning the rapidity of onset and severity of hirsutism, which is usually more pronounced in patients with Cushing syndrome, tumours or non-classic congenital adrenal hyperplasia, is important, as is enquiring about the patient's history of weight gain, hypertension and diabetes. ${ }^{4} \mathrm{~A}$ family history of congenital adrenal hyperplasia can provide a clue to the presence of this disorder.

Patients should also be asked about their current medications, including exogenous steroids (can cause a Cushing phenotype, hyperandrogenism or hyperprolactinemia) and medications that may be used for treatment of the other conditions included in the differential diagnosis.

This information should be used along with the findings from an examination to rule out causes other than polycystic ovary syndrome. These include primarily truncal obesity, purple striae, hypertension (Cushing syndrome), ${ }^{5}$ or signs of virilization including male pattern baldness, deepening of voice and clitoromegaly (rare adrenal tumours). ${ }^{4}$ If these features are noted or the patient's testosterone level is substantially elevated, the patient should be referred to a consultant for further investigation, because additional tests are often complex, nonspecific and insensitive, and require experience in interpreting the results.

\section{What investigations are necessary for diagnosis?}

Investigations to diagnose polycystic ovary syndrome include blood tests for hyperandrogenemia. Calculated free testosterone or free androgen index are recommended options based on the results of a systematic review and an evidence- 
based clinical practice guideline from Australia. ${ }^{1,5}$ Additional androgen measurements are only needed if other causes are suspected based on the patient's history and results of a physical examination. If required, the patient's progesterone level during the luteal phase can be measured to assess ovulatory status. ${ }^{1}$ This test is not required for this patient, because she has substantial oligomenorrhea and ovulatory disturbance.

A pelvic ultrasound can be used to determine the presence of polycystic ovaries and assess endometrial thickness. There is a risk of endometrial hyperplasia in women with oligomenorrhea, although this is uncommon among young women. An ultrasound is not recommended for adolescents because the findings are generally nonspecific (clinical consensus recommendation). ${ }^{1}$

Investigations to exclude other pathology include determining the patient's prolactin and thyroid-stimulating hormone levels. If there is substantial clinical or biochemical evidence of hyperandrogenism, the level of 17-hydroxyprogesterone during the follicular phase should be measured to exclude nonclassic congenital adrenal hyperplasia. ${ }^{1}$

\section{If a diagnosis of polycystic ovary syndrome is made, what other investigations or screening are needed?}

Screening should be undertaken for traditional cardiovascular risk factors, which are increased in women with polycystic ovary syndrome. These include hypertension, dyslipidemia and abnormalities of glucose metabolism. ${ }^{6}$ The following tests are suggested by the Australian guideline, based on clinical consensus. ${ }^{1}$

Body mass index (BMI) should be measured regularly because increased weight exacerbates the reproductive and metabolic features of polycystic ovary syndrome. Monitoring weight facilitates a focus on weight management, with prevention of weight gain as a high priority. Blood pressure should be measured annually or at every visit if the patient's BMI is 25 or greater. In addition, a complete lipid profile should be ordered every two years or annually, if the baseline levels are abnormal or the patient's BMI is 25 or greater. Women with polycystic ovary syndrome often have low high-density lipoprotein cholesterol levels and high triglyceride levels. ${ }^{1}$

Most women (75\%-95\%) with polycystic ovary syndrome have insulin resistance and an increased risk of type 2 diabetes that is independent of, but exacerbated by, obesity. ${ }^{7}$ Insulin resistance cannot be reliably measured in the clinical setting. Because even young and lean women are at risk, all women with polycystic ovary syndrome should be screened for prediabetes and diabetes (clinical consensus recommendation). In particular, these conditions are highly relevant during pregnancy. ${ }^{1}$ Because a fasting glucose test will underdiagnose prediabetes and diabetes, screening should be performed using an oral glucose tolerance test every two years or annually if other risk factors are present (Box 1) (clinical consensus recommendation), ${ }^{1}$ although the frequency of testing remains controversial.

Evidence suggests that there is an increased prevalence of anxiety, depression, eating disorders and psychosexual dysfunction among women with polycystic ovary syndrome; screening for these conditions should be performed using simple validated tools. ${ }^{1}$

\section{What are the implications for fertility?}

Polycystic ovary syndrome is the most common cause of anovulatory infertility and, together with increased BMI, confers increased risks of infertility. ${ }^{1.8}$ Randomized controlled trials (RCTs) have compared various types of lifestyle interventions (incorporating caloric restriction alone, exercise alone, or both) to pharmacologic interventions (metformin or clomiphene) on ovulatory outcomes among women with polycystic ovary syndrome and BMI of 25 or greater. ${ }^{1}$ Although these studies had a high risk of bias, there was no clear difference between lifestyle and pharmacologic therapy. Lifestyle therapy for three to six months is recommended as first-line management for women with a BMI of 30 or greater if there are no other factors affecting fertility. ${ }^{1}$ Weight loss of even 5\% may be sufficient to restore menstrual cyclicity and ovulation. ${ }^{1}$

If a woman is older than 35 years, lifestyle changes have been unsuccessful or her BMI is 30 or greater, pharmacologic therapy should be considered. A recent systematic review of clomiphene compared with metformin (which included the results of two previous systematic reviews and two RCTs) supported the use of clomiphene as first-line treatment for women with anovulatory polycystic ovary syndrome (overall ovulation rate $60 \%-85 \%$; pregnancy rate $30 \%-50 \%$ ). ${ }^{1.9}$

Box 1: Additional risk factors for diabetes in polycystic ovary syndrome ${ }^{1}$

- Age

- Ethnic background

- Parental history of diabetes

- History of high blood glucose levels

- Use of antihypertensive medications

- Smoking

- Physical inactivity

- Waist circumference 
However, there is some evidence from a Cochrane review, which included several RCTs assessing ovulation rate, clinical pregnancy rate, live birth rate and miscarriage, that metformin may be an acceptable first-line treatment for women with a BMI < 32. ${ }^{1,10}$ Metformin can also be added to clomiphene, if there is clomiphene resistance. ${ }^{9}$ The clinical and social circumstances of the couple should be considered when choosing pharmacologic therapy. Clomiphene use has a 5\%-8\% risk of multiple pregnancy and a rare risk of ovarian hyperstimulation. ${ }^{1,9}$

\section{Case revisited}

Given the patient's symptoms (i.e., reported hirsutism and oligo-ovulation), we suspected that she had polycystic ovary syndrome, which was supported by a raised free-androgen index. Her blood pressure, lipid levels and oral glucose tolerance test results were normal, but her BMI was 30. She received education about polycystic ovary syndrome and about the substantial adverse impact of excess body weight on the syndrome, the role of small lifestyle changes to prevent ongoing weight gain, and the benefits of even modest weight loss. In consultation with a dietician, she began a lower caloric-intake diet with healthy food choices and an exercise program of 150 minutes a week, including 90 minutes of aerobic activity at moderate to high intensity. She lost $6.5 \mathrm{~kg}$ ( $8 \%$ of her body weight) over the next 10 months, and her ovulatory cycles resumed.
She has ongoing support to maintain her exercise and prevent weight gain in the longer term.

\section{References}

1. Teede HJ, Misso ML, Moran LJ, et al. Assessment and management of polycystic ovary syndrome: summary of an evidencebased guideline. Med J Aust 2011;195:S65-112.

2. Teede HJ, Joham AE, Paul E, et al. Longitudinal weight gain in women identified with polycystic ovary syndrome: results of an observational study in young women. Obesity (Silver Spring) 2013;21:1526-32

3. Azziz R, Carmino E, Dewailly D, et al. The Androgen Excess and PCOS Society criteria for the polycystic ovary syndrome: the complete task force report. Fertil Steril 2009;91:456-88.

4. Escobar-Morreale HF. Diagnosis and management of hirsutism. Ann N Y Acad Sci 2010;1205:166-74.

5. Guaraldi F, Salvatori R. Cushing syndrome: maybe not so uncommon of an endocrine disease. J Am Board Fam Med 2012; 25:199-208.

6. Rotterdam ESHRE/ASRM-Sponsored PCOS consensus workshop group. Revised 2003 consensus on diagnostic criteria and long-term health risks related to polycystic ovary syndrome (PCOS). Hum Reprod 2004;19:41-7.

7. Stepto NK, Cassar S, Joham AE, et al. Women with polycystic ovary syndrome have intrinsic insulin resistance on euglycaemic-hyperinsulaemic clamp. Hum Reprod 2013;28:777-84.

8. Wild S, Pierpoint T, Jacobs $\mathrm{H}$, et al. Long-term consequences of polycystic ovary syndrome: results of a 31 year follow-up study. Hum Fertil (Camb) 2000;3:101-5.

9. Misso ML, Teede HJ, Hart R, et al. Status of clomphene citrate and metformin for infertility in PCOS. Trends Endocrinol Metab 2012;23:533-43

10. Tang T, Lord JM, Norman RJ, et al. Insulin-sensitising drugs (metformin, rosiglitazone,pioglitazone, D-chiro-inositol) for women with polycystic ovary syndrome, oligo amenorrhoea and subfertility. Cochrane Database Syst Rev 2012;(5(:CD003053.

Affiliation: Monash Centre for Health Research and Implementation, Monash University, Melbourne, Australia

Contributors: Both authors contributed to the writing and revision of the manuscript and approve the final version submitted for publication.

\section{Change of address}

We require 6 to 8 weeks' notice to ensure uninterrupted service. Please send your current mailing label, new address and the effective date of change to:

\section{CMA Member Service Centre}

1870 Alta Vista Dr., Ottawa ON K1G 6R7

tel $888855-2555$ or $613731-8610 \times 2307$

fax 613 236-8864

cmamsc@cma.ca

\section{Changement d'adresse}

II nous faut de 6 à 8 semaines d'avis afin de vous assurer une livraison ininterrompue. Veuillez faire parvenir votre étiquette d'adresse actuelle, votre nouvelle adresse et la date de la prise d'effet du changement, à l'attention du

\section{Centre des services aux membres de l'AMC} 1870, prom. Alta Vista, Ottawa ON K1G 6R7

tél 888 855-2555 ou $613731-8610$ ×2307

fax $613236-8864$

cmamsc@cma.ca

\section{ASSOCIATION MÉDICALE CANADIENNE

